\title{
EXPERIMENTAL SEARCH FOR SUPER AND HYPER HEAVY NUCLEI AT CYCLOTRON INSTITUTE TEXAS A\&M UNIVERSITY*
}
Z. Majka ${ }^{\dagger a}$, M. Barbui ${ }^{b}$, F. Becchetti ${ }^{c}$, G. Chubaryan $^{b}$

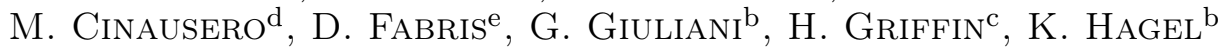
J. KALlunkATHARIYIL ${ }^{\mathrm{a}}$, E-J. Kim ${ }^{\mathrm{b}}$, S. Kowalski ${ }^{\mathrm{f}}$, P. LASKO ${ }^{\mathrm{a}}$ M. Lunardon ${ }^{\mathrm{e}}$, T. Materna ${ }^{\mathrm{b}}, \mathrm{S}^{\mathrm{b}}$ Moretto $^{\mathrm{e}}, \mathrm{R}^{\mathrm{f}}$. Murthy ${ }^{\mathrm{b}}$

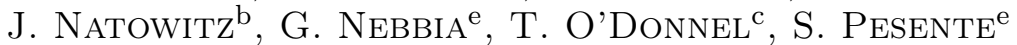

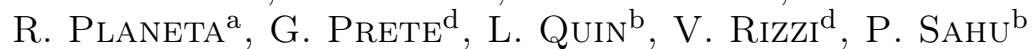

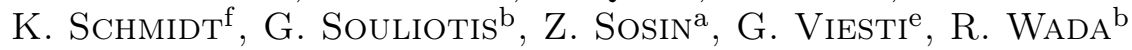

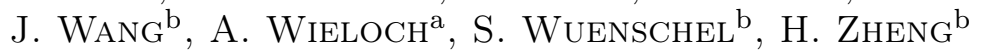

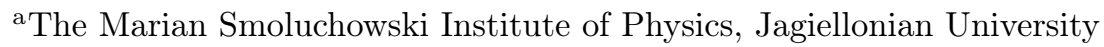
Reymonta 4, 30-059 Kraków, Poland ${ }^{\mathrm{b}}$ Cyclotron Institute, Texas A\&M University, USA ${ }^{\mathrm{c}}$ University of Michigan, Ann Arbor, MI, USA ${ }^{\mathrm{d}}$ INFN Laboratori Nazionali di Legnaro, Legnaro, Italy eDipartimento di Fisica dell'Universitá and INFN Sezione di Padova Padova, Italy ${ }^{\mathrm{f}}$ University of Silesia, Katowice, Poland

\section{(Received December 2, 2013)}

The question "How heavy can an atomic nucleus be?" is a fundamental problem in nuclear physics. The possible existence of island(s) of stable super-heavy nuclei has been an inspiring problem in heavy ion physics for almost four decades. This paper is focused on the experimental search of Super/Hyper Heavy Elements (SHE/HHE) conducted at the Cyclotron Institute, Texas A\&M University. A novel experimental idea and experimental set up introduced for this research will be presented.

DOI:10.5506/APhysPolB.45.279

PACS numbers: 25.70.-z, 25.70.Hi, 25.85.Ge, 29.30.Aj

* Presented at the XXXIII Mazurian Lakes Conference on Physics, Piaski, Poland, September 1-7, 2013.

† zbigniew.majka@uj.edu.pl 


\section{Introduction}

The heaviest known natural element is uranium (U) with the number of protons $Z=92$ in its nucleus. One can also find in natural uranium ores trace quantities of neptunium ( $\mathrm{Np}, Z=93)$, and plutonium $(\mathrm{Pu}, Z=94)$. All elements above $U$ have been produced artificially in heavy ion laboratories across the world and are more or less unstable. The history of the formation of heavy artificial elements begins in the 40s of the former century. In 1934 Enrico Fermi proposed a method to produce such elements. By bombarding a nucleus $(Z, N)$ with neutrons, one obtains a new isotope $(Z, N+1)$ which can beta-decay thus forming a new element $(Z+1, N)$. The first elements created in a laboratory were neptunium and plutonium. They were produced at the University of California in Berkeley in 1940-41. Neptunium was fabricated by McMillan et al. [1] bombarding U with neutrons. Seaborg et al. [2] discovered plutonium-238 through the decay of neptunium-238 which, in turn, were produced by bombarding uranium with deuterons. For the discovery of $\mathrm{Np}$ and $\mathrm{Pu}$ elements E. McMillan and G. Seaborg were awarded Nobel Prize in chemistry in 1951. Elements 99 and 100 were first identified in the debris of the hydrogen bomb test in 1952 (the process reconstructed was the many neutron capture by uranium which then decayed quickly by beta emission to more stable isotopes of elements 99-einsteinium and 100-fermium). To synthesize elements 95, 96, 97, 98 and 101 , it was sufficient to irradiate previously produced heavy nuclei $(93,94$, 99) with neutrons or alpha particles.

In the late 50s, newly constructed accelerators were capable to accelerate heavier nuclei than helium nucleus. These new accelerators opened the door for the creation of even heavier elements known as super-heavy elements (SHE) [3]. Along with the growing number of protons in nucleus, Coulomb repulsion forces cause the decreasing of the fission barrier. When this number becomes large enough, $Z \approx 104$, the barrier should completely vanish, and an instantaneous break up of a nucleus appears. However, Myers and Swiatecki [4] showed in 1966 that closed shells created by quantum effects ensure the existence of the barrier even for nuclei with $Z>114$. Further theoretical elaborations, based on the shell model, predicted more precisely that the next closed shells should emerge for spherical nuclei at $Z=114$ and $N=184$ (neutron number). Such nuclei would be located in the center of the island of stability of super-heavy elements. Their half-life times, with respect to the spontaneous fission were estimated from a few years to many thousands of years. The calculations also revealed, that alpha radioactivity is the main decay mode of those nuclei [5] and one should expect an increase of their half-life times for this mode of decay. Experiments targeted on reaching this hypothetical island of stability were initiated. Production of the super-heavy elements, $Z>104$, was accomplished by complete fusion reactions in nuclear collisions by laboratories operating heavy ion acceler- 
ators: Joint Institute for Nuclear Research (JINR) in Dubna, Gesellschaft fuer Schwerionenforschung (GSI) in Darmstadt, Lawrence Berkeley National Laboratory (LBNL) in Berkeley. Such studies were possible because of the impressive progress made in accelerator technology, and new, highly efficient heavy ion sources. By the year 1996, elements with $Z$ up to 112 were discovered in this way [6-9]. Unfortunately, during those studies it turned out that the cross section for super-heavy nuclei production in fusion reactions is decreasing quite rapidly: with every next $Z$ more or less by factor 4 reaching for element with $Z=112$ about $1 \mathrm{pb}$. This was a very serious limitation in the synthesis of the next elements. Moreover, half-life times of the most heaviest ones were becoming as short as a few tens of $\mu$ s. Such small values of half-lives has shaken the belief in the existence of the island of stability for SHE. One of the possible explanations for these results was that the newly produced elements were highly neutron deficient isotopes, and they should, in fact, have quite short lifetimes. Simply available combinations of projectiles and targets could not be used to produce more neutron-rich nuclei. At present, the periodic table contains elements with atomic number $(Z)$ up to 118. The most recent discoveries (1998-2005) made by the DubnaLivermore Collaboration, a synthesis of elements $Z=114$ in the reaction ${ }^{48} \mathrm{Ca}+{ }^{242,244} \mathrm{Pu}, Z=116$ (year 2000) in the reaction ${ }^{48} \mathrm{Ca}+{ }^{248} \mathrm{Cm}$ [10], and $Z=118$ (year 2002) in the reaction ${ }^{48} \mathrm{Ca}+{ }^{249} \mathrm{Cf}$ [11-13], which still need to be confirmed by other laboratories, delivered a new impulse to search for the next heavy elements, and to synthesize new isotopes of already known elements. In the case of both reactions, the half-life times of produced nuclei, for $Z=114$ is a few seconds, indicating an increase in stability. This brings new hope for the existence of the island of stability in the region predicted by the theory: $Z=114, N=184$.

In fact, all artificial elements beyond fermium were created by complete fusion of heavy ions. Two types of approaches have been used in this case: the "cold" fusion with bismuth or lead targets and projectiles of most neutron rich isotopes like ${ }^{64} \mathrm{Ni}$ or ${ }^{70} \mathrm{Zn}$ to produce elements 110 and 112 , and "hot" fusion of actinide targets such as $\mathrm{Pu}, \mathrm{Cm}$ or $\mathrm{Cf}$ with ${ }^{48} \mathrm{Ca}$ projectiles to reach elements with $Z=114,116,118$ [14]. In the case of "cold" fusion reactions, the created super-heavy nuclei possess low excitation energy $E *=10-15 \mathrm{MeV}$, while for "hot" reactions excitation energy is $E *=30-40 \mathrm{MeV}$. In both cases, only some of them can survive as a residue and reach their ground state or isometric states by 1-2, or 3-4 neutrons emission depending on whether the reaction studied is "cold" or "hot". Most disintegrate immediately because the fission barrier is nearly equal, or even lower than the neutron binding energy. As a result, the cross section for residue creation is a product of a cross section for fusion reaction and probability, that in a cooling phase, fission will not occur. 
A very recent publication [15] reports that element 115 was produced independently by a second group and this element is waiting for naming.

The question "How heavy can an atomic nucleus be?" is still not answered and the location of the stability island of super-heavy elements is not yet experimentally determined.

\section{Concept of the SHE creation}

All artificial elements beyond fermium were created by complete fusion of heavy ions. It is well known that the cross sections for super-heavy nuclei production are extremely low $(\sigma \approx 1 \mathrm{pb}$ for synthesis of $Z=112$ nucleus and $\sigma \approx 0.5 \mathrm{pb}$ for synthesis of $Z=118$ nucleus) [13, 16]. Therefore, the experiments dedicated to super-heavy nuclei synthesis require a large amount of the accelerator beam time. For even heavier nuclei, one can expect that the cross section is dropping into the region of tens of fb. This creates a serious limitation for the technique being used so far. A completely new generation of heavy ion sources is needed to supply the intensity of ion beams as high as $10^{14-15}$ particles/sec. Moreover, the available combinations of the neutron to proton ratio of the projectiles and targets are very limited, and it is difficult to be optimistic about the possibility to reach the island of stability of super-heavy elements using complete fusion reactions with a stable projectiles.

In this context, the collaboration of scientists from the Cyclotron Institute Texas A\&M University, the Institute of Physics of the Jagiellonian University, and the Instituto Nazionale di Fisica Nucleare di Legnaro have used since 2002 the heavy nuclei reactions for the SHE creation and search [17]. The idea is visualized in Fig. 1 where nuclear reactions induced at low incident energies by heavy ion projectiles $\left(\right.$ e.g. $\left.{ }^{172} \mathrm{Yb},{ }^{197} \mathrm{Au}\right)$ on fissile target nuclei (e.g. $\left.{ }^{232} \mathrm{Th},{ }^{238} \mathrm{U}\right)$ are schematically illustrated.

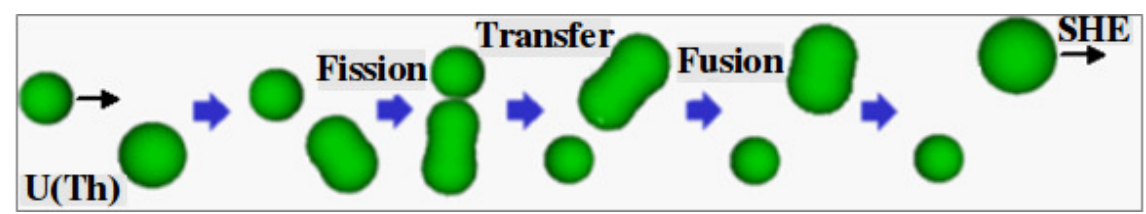

Fig. 1. Schematic view of the possible reaction between the fissile target nucleus $\mathrm{U}$ $(\mathrm{Th})$ and a heavy projectile (e.g. $\mathrm{Yb}, \mathrm{Au})$.

One possible scenario for such reactions is depicted in the figure. In the initial stage of the collision, a heavy projectile approaches the target nucleus initiating deformation process of the latter one (two first snapshots in the figure). Next, nuclear interactions take place between the objects (third snapshot in the figure) for a period long enough to transfer a large 
amount of mass (by fission or massive transfer processes). Finally, if the fusion conditions are met during the transfer process, e.g. small relative angular momenta of both merging masses, an excited very heavy system can be formed (last two snapshots in the figure). Does the system survive as a ground state SHE nucleus (residue evaporation)? This depends on the excitation energy and angular momentum brought into this system during the process - the lower the excitation energy and angular momentum the larger the chance that the system will end up as an evaporation residue.

Certainly, the probability of that scenario is very small but there are several attractive features in such a scenario. It is well known that nuclear products originating from low-energy nuclear fission are characterized by broad spectra of mass and atomic number, and the majority of them are neutron-reach species (fission fragments). If such a species is transferred to the projectile nucleus, as in the proposed collision scenario, the fusion probability can be strongly enhanced due to its neutron excess. On the other hand, in a situation when the fragment brought to the projectile is not neutron-rich, but has a proton/neutron number near the closed shells, then the survival probability of the formed super-heavy nucleus will increase as the fission barrier of the resulting SHE nucleus is high. Besides, if the transfer process of the fission (or massive) fragment takes place at the peripheral collision, the resulting system should be formed with small excitation energy. In consequence, its survival probability again increases. Both factors: fusion probability and survival probability play a decisive role in the production of the SHE elements because they determine the residue cross section.

Although model calculations that estimate the value of the residue cross section in such a mechanism are scarce, we know that the fissile nuclei have a very high fission cross section thus one can conjecture that the SHE elements might be produced here with probabilities that are competitive compared to the classical "hot" or "cold" fusion reactions. In addition, the fission of target nuclei is a kind of "ion source" (in fact, this is the way the physicists that are presently developing radioactive ion beams treat the fission of uranium) that delivers a wide spectrum of different heavy ions, therefore, "nature itself can choose" the most appropriate one that will fuse with the projectile ion in order to produce a super-heavy nucleus. Using, for example, heavy ion projectile $Z=79(\mathrm{Au})$ and the target nucleus $Z=90(\mathrm{Th})$, we may be able to explore properties of super-heavy and possibly hyper-heavy nuclear systems in the regions of atomic numbers $Z \approx 112$ and $Z \approx 136$, keeping in mind that the low-energy fission of thorium produces a two humped atomic number spectrum of fission fragments, one hump is peaked at $Z=33$ and the other at $Z=57$. In these reactions, as in fusion reactions, shell effects might be an important factor. For heavy nuclei shell effects can play an important role in determining potential energy surfaces, fusion probabilities, 
decay probabilities and fragmentation patterns as long as the excitation energy is not too high. This premise is supported by recent calculations of Zagrebaev et al. $[18,19]$ who have begun to explore theoretically the production of new heavy isotopes using damped collisions of very heavy nuclei. They point out that the role of the shell effects in damped collisions of heavy nuclei is still not absolutely clear and was not carefully studied.

There is also a drawback of the method. In the proposed reactions, a relatively long interaction time is needed to transfer the target fission (or massive) fragment to the projectile. This implies that the deep inelastic collisions will also be involved in the transfer process. As a result, quite high excitation energy of the reaction partners can be generated and consequently the survival chance of the produced super-heavy nucleus can decrease dramatically. On the other hand, the second fission (or massive) fragment can be considered a heat and angular momentum reservoir with respect to the rest of the system. If it absorbs the main part of the dissipated kinetic energy of the collision, the super-heavy species will leave the reaction region with low excitation and its survival probability will be high.

\section{The first experimental realization}

Experimental instruments that are located in the Cyclotron Institute of TAMU are well suited for the proposed experiments. The K500 cyclotron delivers reasonable intensity beams of heavy ions up to uranium. The energies of the beams are in the range of interest: from $\sim 5$ to $15 \mathrm{AMeV}$. Moreover, an efficient magnetic velocity filter was available at the time when our research plan was born. This filter, the superconducting solenoid (BigSol) constructed at the Michigan State University [17], is characterized by a strong magnetic field and a large entrance angular acceptance. Because interesting reaction products might be emitted at angles greater than $\sim 1$ degree, the velocity filter technique allows separation of those products from other heavy ions produced in different reaction channels.

The objective of a few test measurements was to verify experimentally the idea presented in the last section, especially to estimate the cross section for SHE production and to test whether the method can be competitive with the standard complete fusion approach. Also a few novel detection and identification methods of produced heavy ions were investigated.

The experience that we gained in the first set of test measurements has been utilized in the experiment conducted in August 2006. The experimental set up constructed for this measurement is presented in Fig. 2. 


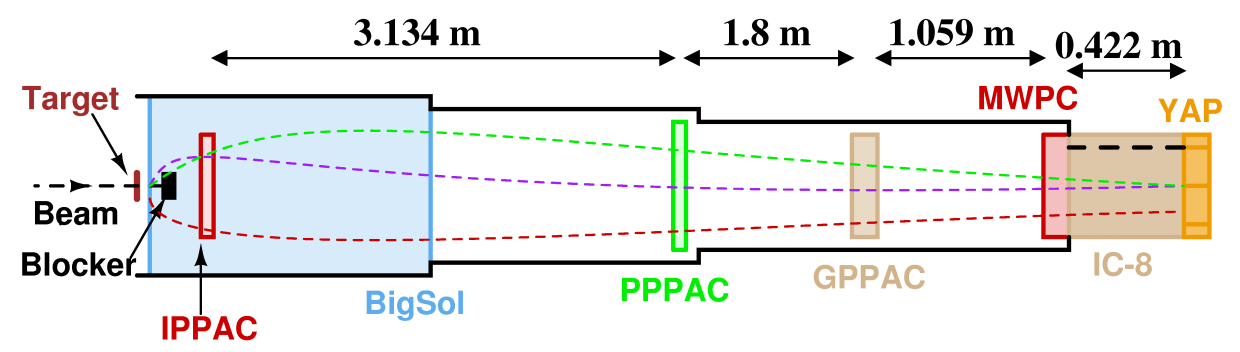

Fig. 2. Momentum and charge filter (BigSol) and the detection system used in the first set of test experiments at the TAMU University (see the text for details).

The reaction ${ }^{197} \mathrm{Au}(7.5 \mathrm{AMeV})+{ }^{232} \mathrm{Th}$ was selected as the most suitable to produce the super-heavy systems. The search for very rare events sets extraordinary demands an experimental set up and off-line analyses. The beam is stopped by the "Blocker" which also diminishes projectile-like fragments emitted at small angles. It is also shown how the BigSol magnetic field separates trajectories of different heavy ions at the exit of the BigSol, trajectories have different distances from the beam axis (PPPAC is the detector build in Kraków). SHE nuclei are "implanted" in the ionization chamber equipped with 8 segments (IC-8), each of the segments measure energy loss of super-heavy nuclei. The detectors GPPAC and PPPAC, both of PPAC type detectors, are used for time-of-flight (ToF) measurements together with MPWC chamber which gives a very fast time signal.

The collected statistics contained 4 million events and the preliminary results [20] presented in Fig. 3 indicate that three events were identified as very heavy ions.

The size of this article limits detailed presentation of experimental procedure and off-line analysis. The arguments that support the hypothesis that three recorded events are very heavy ions are:

- Pulse shape distribution in the IC-8 is very different from that of the beam-like ions: much higher amplitude in the first segment of the IC-8 and the decrease rate in its consecutive segments is also much higher than those of beam-like ions.

- Measured velocities between PPPAC-GPPAC and between GPPACMWPC are consistent with the detection of a single ion in the event.

- The FWHM of the pulse shape is also consistent with the hypotheses that a single ion was detected in each of those three events because the beam-like particles have very similar FWHM. 


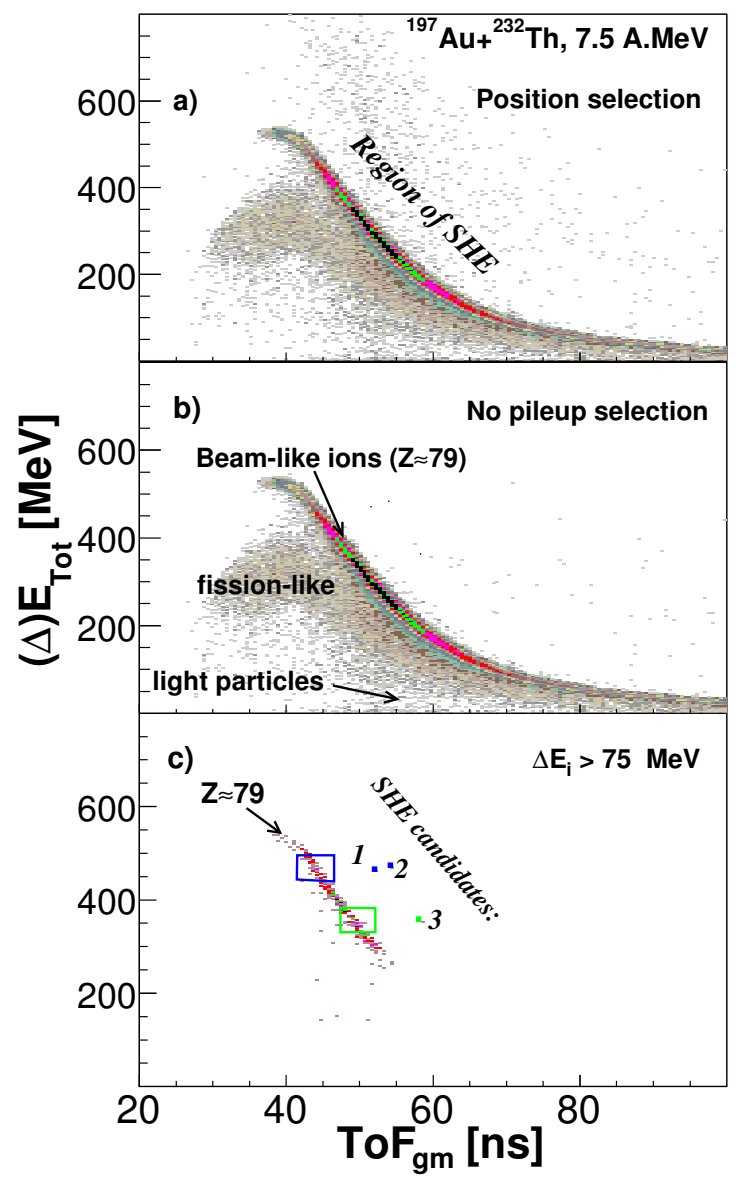

Fig. 3. Total energy loss $\left(\sum_{i} \Delta E_{i}, i=1, \ldots, 8\right)$ in the ionization chamber of detected heavy ions versus their ToF between detectors GPPAC-MWPC; (a) represents events when the heavy ions deliver proper position signals in the ToF detectors: GPPAC and MWPC, (b) requirement that no pileup is present was added, (c) additionally, we required that the $\Delta E_{i}$ of the detected heavy ion in one of the segments of the IC- 8 is greater than $75 \mathrm{MeV}$. In (c), three events are isolated in the region of the expected SHE nuclei [20].

This preliminary results were revisited, at a later stage of data analysis, and looking in more detail at these interesting events, we concluded that only one of them can be assigned to the detection (production) of very heavy nucleus with the atomic number in the range of $Z=100-110$ (the approximate estimation of $Z$ is dictated by the identification capabilities of the IC-8 itself). Nevertheless, the result is important as it indicates that our experimental approach is capable to produce and detect very heavy nuclei. 
The conclusion of this set of test experiments can be stated as follows:

- The reaction ${ }^{197} \mathrm{Au}+{ }^{232} \mathrm{Th}$ at $7.5 \mathrm{AMeV}$ was investigated at Texas A\&M using the BigSol as a velocity spectrometer for SHE production.

- A large mass transfer is observed in the detected reaction products atomic number larger than 79 (rise up to $5 Z$ ).

- The cross section for those events is about $55 \mathrm{nb}$. The $Z$ resolution of the system for those events is about $10 \%$ because their energy is very much degraded before the IC. Therefore, a precise identification of the nuclei was not possible.

- Further improvements of the experimental set up were postulated in order to reduce the energy loss of the ions in the detectors. A higher granularity of the IC is also recommended in order to improve the efficiency of the pileup rejection.

In summary, the results show a possible way to produce nuclei with $Z$ above 100 via heavy mass transfer scenario.

Unfortunately, the experiment with this experimental set up was discontinued due to a spectrometer He leak.

\section{A new experimental set up}

The collected experiences and the positive experimental result justify continuation of this unique and promising research program. However, a successful realization of this ambitious plan requires a new concept for detection and identification of produced super-heavy nuclei. In 2012, a new active catcher detection system was proposed for SHE search. The active catcher detection system was constructed basing on the experiences obtained during a set of test experiments with passive catcher experimental set up where an idea to identify production of SHE via characteristic alpha decay was positively tested. Many super-heavy elements are expected to decay by alpha emission with unusually high energies (see e.g. [21]) and this observation delivers a method to distinguish such decay from that of lighter elements.

The idea of the new experimental approach to the identification of SHEs created in the alternative reaction mechanism described in Section 2 (massive transfer e.g. in the ${ }^{197} \mathrm{Au}+{ }^{232} \mathrm{Th}$ reaction) involves two phases of the reaction products detection and identification. In the first phase, the heavy reaction products are implanted and registered in the active catcher detection element (see Fig. 4). 


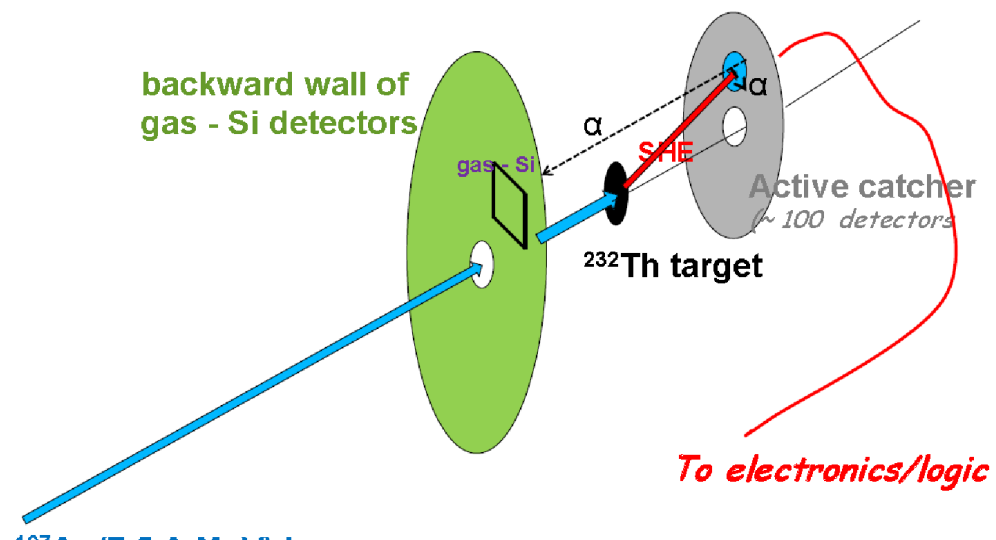

${ }^{197} \mathrm{Au}(7.5 \mathrm{~A} . \mathrm{MeV})$ beam

Fig. 4. Active catcher detection system (see the text).

In the second phase, a characteristic alpha particle decay of the product deposited in the active catcher detector is detected in the backward hemisphere by $\Delta E$ (gas) $E(\mathrm{Si})$ telescopes or by the active catcher detection segment. (Spontaneous fission decays are also detectable in the active catcher.) The active catcher detection segment presented in Fig. 5 consists of the fast plastic scintillator, where the ${ }^{197} \mathrm{Au}+{ }^{232} \mathrm{Th}$ reaction products are implanted, an aluminium cylinder with a parabolic cavity which serves as a light guide, and the photomultiplier tube (Hamamatsu, $\Phi=8 \mathrm{~mm}$ ). The signals from the active catcher detection segment are analyzed by dedicated electronics.

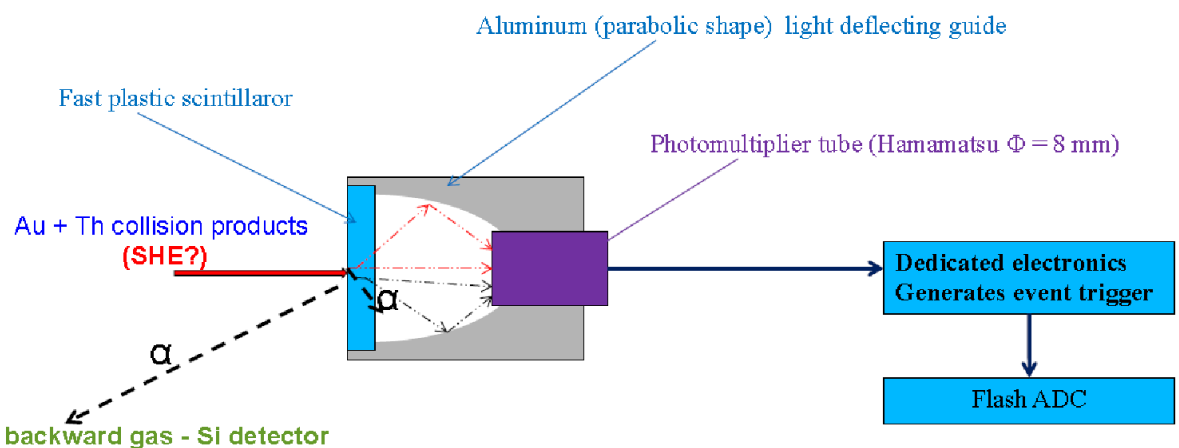

Fig. 5. Schematic drawing of the active catcher detection segment (see the text).

The test runs to verify the method were investigated experimentally with a passive catcher detection set up (the reaction products were implanted into polypropylene sheets placed in the position of active catcher in Fig. 4). The 
results presented in Fig. 6 show observation of signals in the backward wall of detectors which might be assigned to high energy particles. However, in this simple experimental set up, it was impossible to make all correlations necessary to characterize the emitter. It should be mentioned that a very proton rich nuclei can produce higher energy alphas as can ternary fission. This has to be further explored to make a convincing case.
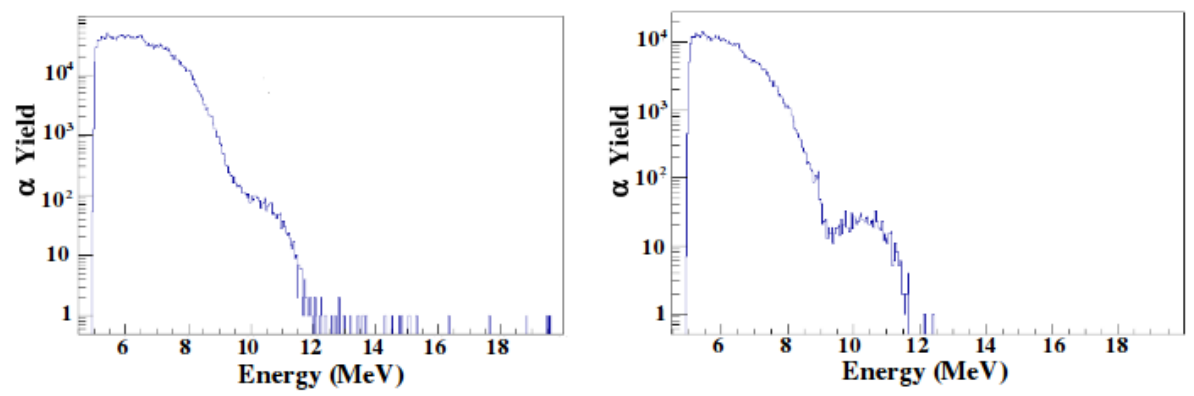

Fig. 6. Observed alpha particle decay energy distributions, beam-on (left) and beam-off (right) detected by backward hemisphere Si wall during test run with passive catcher detection set up.

Elements of the active catcher detection system presented in Fig. 4 were used for the first time in August 2013 in a test experiment at the Cyclotron Institute, Texas A\&M University. Our analysis of the collected data is in progress and very preliminary results show:

- The active catcher detection system has a very good time resolution (scintillation pulse width is about $5 \mathrm{~ns}$ and their rise times $\sim 1 \mathrm{~ns}$ ).

- The dynamical range allows to distinguish between deposited heavy reaction products and alpha particles.

- The observation in passive catcher test experiments of high energy alpha particles was also confirmed in this measurement.

- Further improvements of the experimental set up are underway in order to improve event selection.

The next measurements are scheduled for the early part of 2014 .

This work is supported by the National Science Center in Poland, contract No. UMO-2012/04/A/ST2/00082, by the U.S. Department of Energy under Grant No. DE-FG03-93ER40773 and by the Robert A. Welch Foundation under Grant A0330. 


\section{REFERENCES}

[1] E.M. McMillan, P.H. Abelson, Phys. Rev. 57, 1185 (1940).

[2] G.T. Seaborg, E.M. McMillan, J.W. Kennedy, A.C. Wahl, Phys. Rev. 69, 366 (1946).

[3] See e.g. K. Kumar, Super Heavy Elements, Adam Hilger, Bristol and New York 1989.

[4] W.D. Myers, W.J. Swiatecki, Nucl. Phys. 81, 1 (1966).

[5] A. Sobiczewski, F.A. Gareev, B.N. Kalinkin, Phys. Lett. 22, 500 (1966).

[6] S. Hofmann, G. Münzenberg, Rev. Mod. Phys. 72, 733 (2000).

[7] H. Hofmann, R. Samhammer, G. Ockenfuss, Nucl. Phys. A496, 269 (1989).

[8] S. Hofmann et al., Z. Phys. A350, 281 (1995).

[9] S. Hofmann et al., Z. Phys. A354, 229 (1996).

[10] Yu.Ts. Oganessian, JNRS 3, 5 (2002).

[11] Yu. Oganessian, JINR Preprints and Communications, Dubna, D7-2002, 287 (2002).

[12] Yu. Oganessian, LNL Report, Berkeley, UCRL-ID-151619, (2003).

[13] Yu. Oganessian, Phys. Rev. C74, 044602 (2006).

[14] Yu. Oganessian, Phys. Rev. C63, 011301 (2001).

[15] D. Rudolph et al., Phys. Rev. Lett. 111, 112502 (2013).

[16] S. Hofmann et al., Eur. Phys. J. A14, 147 (2002).

[17] T. Materna et al., Progress in Research, April 1, 2003-March 31, 2004, Cyclotron Institute Annual Reports, Texas A\&M University, pp. II-17, 2004.

[18] V.I. Zagrebaev et al., J. Phys.: Conf. Series 420, 012001 (2013).

[19] V.I. Zagrebaev, W. Greiner, Phys. Rev. C87, 034608 (2013).

[20] M. Barbui et al., Int. J. Mod. Phys. E18, 1036 (2009).

[21] M. Bender, P.-H. Heenen, J. Phys.: Conf. Series 420, 012002 (2013). 